\title{
Identification of an insecticidal crystal protein from Bacillus thuringiensis DSIR517 with significant sequence differences from previously described toxins
}

\author{
Andrew P. Gleave, ${ }^{*}$ Rebecca J. Hedges and Andrew H. Broadwell \\ Molecular Biology Group, DSIR Plant Protection, Private Bag, Auckland, New Zealand
}

(Received 28 June 1991; revised 17 September 1991; accepted 11 October 1991)

\begin{abstract}
The nucleotide (nt) sequence of a DNA segment containing the majority of a gene cloned from Bacillus thuringiensis DSIR517 encoding a $130 \mathrm{kDa}$ insecticidal crystal protein has been determined. Sequence analysis reveals an open reading frame (ORF) of $3453 \mathrm{nt}$. The ATG initiation codon, which is preceded by a potential ribosome-binding site sequence, was confirmed by $\mathrm{N}$-terminal amino acid sequencing. The ORF extends beyond the $3^{\prime}$ terminus of the cloned fragment; however, the high degree of homology between the deduced amino acid sequence of this ORF and other Cry proteins suggests the clone lacks only five C-terminal amino acids. Making this assumption, the ORF of $3468 \mathrm{nt}$ encodes a protein of 1156 amino acids with an estimated molecular mass of 129700 Da. Analysis of the deduced amino acid sequence reveals a number of features characteristic of Cry proteins. Alignment of the Cry 517 protein sequence with other Cry proteins suggests it is most closely related to the $\operatorname{cry} I A-E$ genes but sufficiently different to form a new cryI gene subclass.
\end{abstract}

\section{Introduction}

Bacillus thuringiensis is a Gram-positive soil bacterium characterized by the production of proteinaceous crystals during sporulation. The crystals are composed of proteins which exhibit highly specific insecticidal activity. B. thuringiensis strains active against larvae of certain members of Lepidoptera, Diptera or Coleoptera have been identified and over 40 cry genes encoding insecticidal crystal proteins (ICPs), active against these three insect orders, have been cloned and sequenced. These cry genes are classified according to their nucleotide sequence and the host specificity of their product (Hofte \& Whiteley, 1989). Genes encoding lepidopteran-active proteins $(130-140 \mathrm{kDa})$ are designated as $c r y I$, as is a $c r y$ gene of $B$. thuringiensis subsp. aizawai IC-1 with lepidopteran and dipteran activity. Similarly, genes encoding lepidopteran-active and lepidopteran/dipteran-active proteins $(65 \mathrm{kDa})$ are desig-

* Author for correspondence. Tel. (09) 893660 ; fax (09) 863330.

Abbreviations: ICP, insecticidal crystal protein; nt, nucleotide(s); RBS, ribosome-binding site.

The nucleotide sequence data reported in this paper have been submitted to Genbank and have been assigned the accession number X58534. nated as $c r y I I$, coleopteran-active proteins $(73 \mathrm{kDa})$ as cryIII and dipteran-active proteins $(72-135 \mathrm{kDa})$ as crylV.

There have been a number of reports of insect resistance to $B$. thuringiensis ICPs (McGaughey, 1985; McGaughey \& Beeman, 1988; Tabashnik et al., 1990). In one instance, such resistance was attributed to a reduction in binding affinity of the proteolytically activated toxin to the membrane of the susceptible insect epithelial midgut cells (Van Rie et al., 1990). Binding of the toxin is necessary for subsequent cell lysis (Wolfersberger, 1990), which results in the death of the larva.

As part of a strategy to minimize the potential problems of resistance to $B$. thuringiensis ICPs we have initiated a programme to identify novel $B$. thuringiensis cry genes, with the aim of obtaining new proteins which bind to alternative membrane receptors on the insect midgut cells. Increasing the diversity of genes a vailable, for either microbial insecticides or transgenic plants, would facilitate the simultaneous use of genes encoding insecticidal proteins which bind to different membrane receptors, and should both increase the efficiency of pest control and delay the emergence of resistance.

In this report we describe the determination of the nucleotide sequence of a novel $c r y$ gene cloned from $B$. thuringiensis DSIR517, isolated from an agricultural soil sample in New Zealand (Wigley \& Chilcott, 1990). B. 
thuringiensis DSIR 517 produces crystals, composed of at least two $130-135 \mathrm{kDa}$ proteins and a $60 \mathrm{kDa}$ protein, which are toxic to the leafroller Epiphyas postvittana (Lepidoptera: Tortricidae), a major horticultural pest in New Zealand. The deduced amino acid sequence, encoded by the cloned gene, is compared with that of previously reported Cry proteins and discussed.

\section{Methods}

Bacterial strains and culture conditions. Bacillus thuringiensis DSIR517 was obtained from the DSIR B. thuringiensis culture collection. E. coli JM109 (Yanisch-Perron et al., 1985) was used as the host for the Bluescript SK ${ }^{+}$(Stratagene) and pGEM7Zf- (Promega) derivatives. JM109 harbouring these derivatives were grown at $37^{\circ} \mathrm{C}$ in LuriaBertani medium (Maniatis et al., 1982) containing ampicillin $\left(50 \mu \mathrm{g} \mathrm{m}^{-1}\right)$. Agar was added to $1 \cdot 5 \%(\mathrm{w} / \mathrm{v})$ to solidify the medium.

Plasmids and phage. Plasmids pSK517 and pGEM517, derivatives of Bluescript $\mathrm{SK}^{+}$and $\mathrm{pGEM} 7 \mathrm{Zf}^{-}$, respectively, containing a $5.2 \mathrm{~kb}$ $E c o$ RI fragment encoding most of a cry gene from $B$. thuringiensis DSIR517 (A. H. Broadwell and coworkers, unpublished) were used as the source of DNA in nucleotide sequence determination. Plasmid DNA was isolated as described by Del Sal et al. (1988). Single-stranded template DNA for nucleotide sequencing was prepared essentially as described in the Stratagene sequencing manual, using R408 helper phage.

DNA manipulations. Transformation of $E$. coli was carried out using a modified procedure of Meyer et al. (1977). Restriction enzymes and T4 DNA ligase (Bethesda Research Laboratories, Boehringer Mannheim, New England Biolabs) were used according to the manufacturers recommendations. Exonuclease III/SI nuclease unidirectional deletions were generated as described for the Erase-a-Base System (Promega) with minor modifications. All other recombinant DNA techniques were as described in Maniatis et al. (1982).

Protein analysis and sequencing. Crystals of $B$. thuringiensis DSIR517 were purified by growing the cells to sporulation at $30^{\circ} \mathrm{C}$ in $\mathrm{CCY}$ media (Stewart et al., 1981). Spores and crystals were washed in $1 \mathrm{M}$ $\mathrm{NaCl} / 10 \mathrm{~mm}$-EDTA and separated by differential centrifugation through sucrose gradients. Polypeptides of purified crystals were separated by $5 \%(\mathrm{w} / \mathrm{v})$ polyacrylamide gel electrophoresis using a Hoefer SE600 gel system. Polypeptides were electroblotted onto polyvinylidene difluoride membranes as described by Matsudaira (1987) and subjected to automated Edman degradation amino acid sequencing in an Applied Biosystems Gas Phase Sequenator (Model 470A). Recombinant protein preparations from $\lambda g t l l$ lysogens, immunological screening of plaques and Western blotting were all carried out as described in the Promega Protocols and Applications Manual.

Nucleotide sequencing. Dideoxy chain termination sequencing (Sanger et al., 1977) of single-stranded DNA templates was carried out using a Sequenase kit (United States Biochemical Corporation) incorporating $\left[\alpha^{-35} \mathrm{~S}\right] \mathrm{dATP}$ (Amersham International). The universal 17-mer primer and custom-made 17-mer oligonucleotides synthesized on a Cyclone Plus DNA Synthesizer (Milligen) were used as primers. Both strands of the cry-517 ORF were sequenced.

Nucleotide and amino acid sequence analysis. Sequence data analysis was carried out using the LINEUP, BESTFIT, GAP and GAPSHOW version 5 programs of the University of Wisconsin, Genetics Computer Group Sequence Analysis Package, on a MicroVAX 3400 computer.

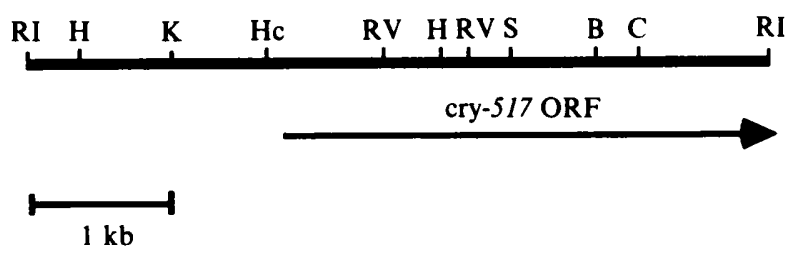

Fig. 1. Restriction map of $5.2 \mathrm{~kb} E c o$ RI fragment cloned from $B$. thuringiensis DSIR 517. The position of the cry-517 ORF and direction of transcription are indicated by the arrow. Restriction sites: B, BamHI; C, ClaI ; H, HindIII; Hc, HincII; K, KpnI; RI, EcoRI; RV, EcoRV; S, SstI.

\section{Results and Discussion}

\section{Nucleotide sequence of the cry-517 gene}

Total genomic DNA, isolated from $B$. thuringiensis DSIR517, was digested with EcoRI and ligated to the $E c o R I$ arms of $\lambda g t 11$. Recombinant $\lambda g t 11$ plaques were screened immunologically with antibody raised against purified crystals of $\boldsymbol{B}$. thuringiensis DSIR517, to identify clones expressing a cry gene. Restriction mapping of positive clones indicated that they all contained a $5 \cdot 2 \mathrm{~kb}$ EcoRI insert. To facilitate the determination of the nucleotide sequence of the $c r y$ gene cloned from $B$. thuringiensis DSIR517, the $5 \cdot 2 \mathrm{~kb}$ EcoRI fragment (Fig. 1) was subcloned into the Bluescript vector $\mathrm{SK}^{+}$ (Stratagene) and pGEM7Zf- (Promega), generating pSK517 and pGEM517, respectively. Exonuclease III/SI nuclease deletions were generated across the $5 \cdot 2 \mathrm{~kb}$ insert in both directions and single-stranded templates of suitable clones were sequenced.

The nucleotide sequence of the cloned cry-517 gene from B. thuringiensis DSIR517 consists of a protein coding region of $3453 \mathrm{nt}$, as shown in Fig. 2. The ATG initiation codon is located at nt 385 , preceded by a potential RBS, 5'-GTAGGAGGAAAA-3'. Confirmation of the proposed initiation codon was established by Edman degradation amino acid sequencing, which indicated the five $\mathrm{N}$-terminal amino acids of the $130 \mathrm{kDa}$ protein of $B$. thuringiensis DSIR517 to be M-N-Q-N-K.

Sequence analysis reveals that the $3^{\prime}$ terminus of the cry-517 ORF lies beyond the end of the cloned fragment. The high degree of homology between the C-termini of the protein encoded by the cloned gene and those of other Cry I proteins implies that the clone lacks five C-terminal amino acids. Assuming that the clone lacks these 5 amino acids, the ORF (3468 nt) codes for a protein of approximately $129.7 \mathrm{kDa}$ consisting of 1156 amino acid residues. This is consistent with the size of the protein as estimated by SDS-polyacrylamide gel electrophoresis (130 kDa). 


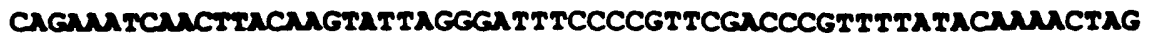

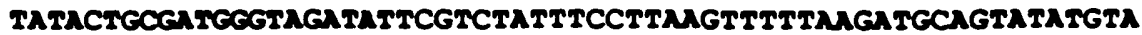

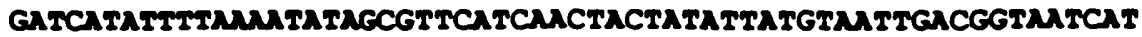

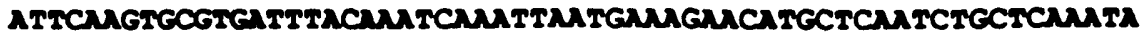

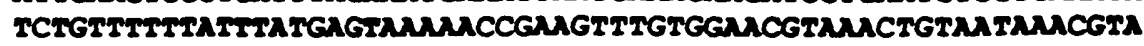

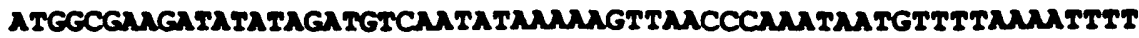
МNAтגА $\begin{array}{llllllllllll}4 & N & Q & 8 & K & A & G & I & I & G & A & s\end{array}$

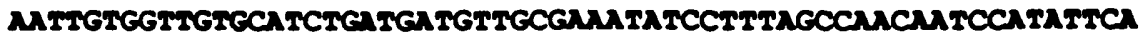

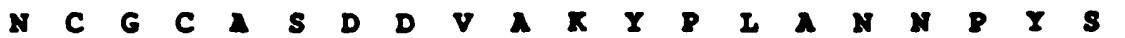

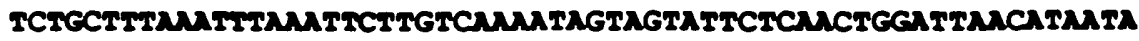

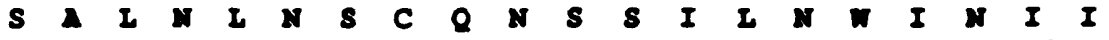

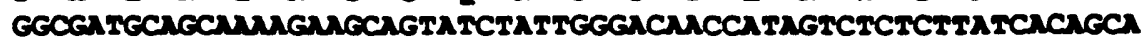
$G \begin{array}{lllllllllllllllllll}G & D & A & A & I & A & V & S & I & G & T & I & I & V & s & I & I & I & A\end{array}$

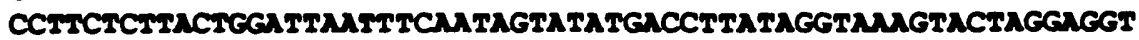
$\begin{array}{llllllllllllllllllll}P & 8 & I & I & G & I & I & S & I & V & Y & D & I & I & G & K & V & I & G & G\end{array}$

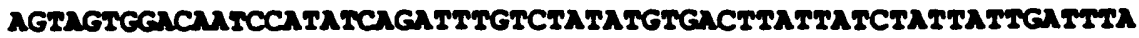
$\begin{array}{llllllllllllllllllll}5 & 8 & G & 9 & 5 & I & S & D & I & S & I & C & D & I & I & s & I & I & D & I\end{array}$

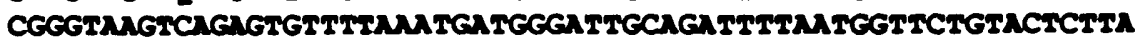
$\begin{array}{llllllllllllllllllll}R & V & S & Q & S & V & I & N & D & G & I & A & D & I & N & G & 8 & V & I & I\end{array}$

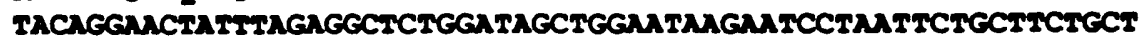

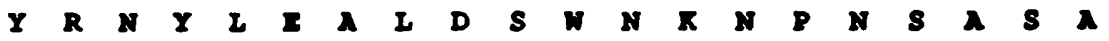

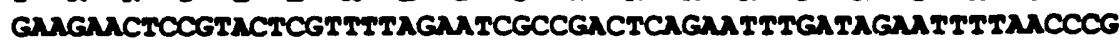

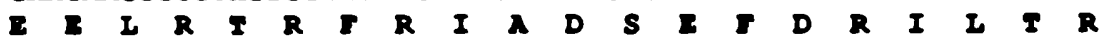

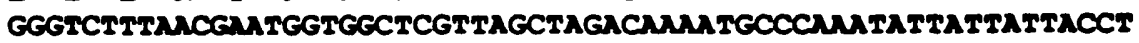

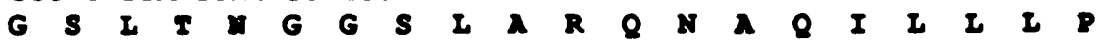

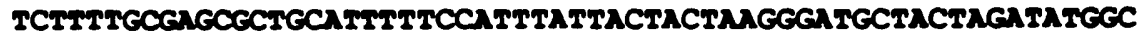

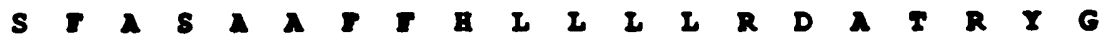

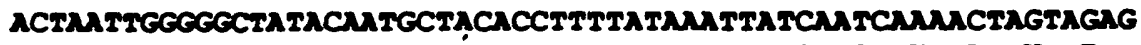
I I $G$ I $Y$ \& $A$ I $P$ I I $Y$ Y $Q$ S $X$ I V I

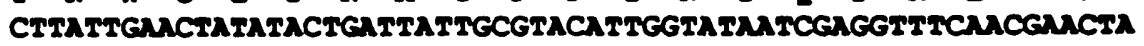
$\begin{array}{llllllllllllllllllll}I & I & I & I & I & I & D & Y & C & V & A & V & Y & N & R & G & I & I & I & I\end{array}$

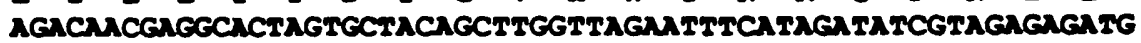

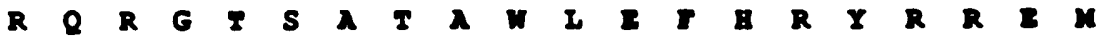

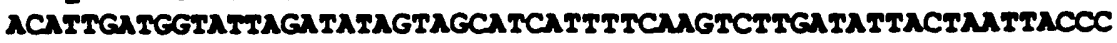

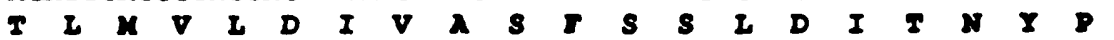

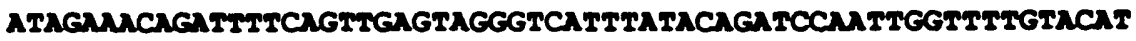

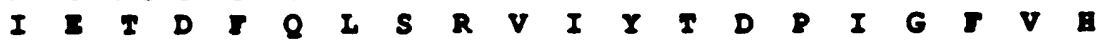

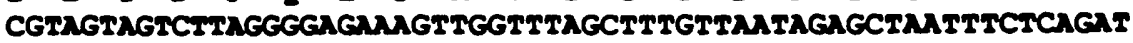

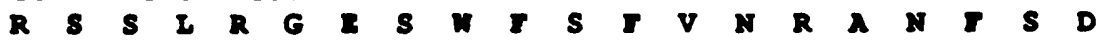

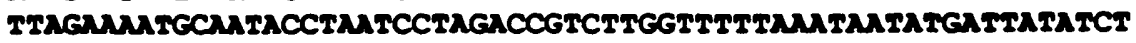
$\begin{array}{llllllllllllllllllll}I & I & A & A & I & P & A & P & R & P & S & W & I & I & A & y & A & I & I & S\end{array}$

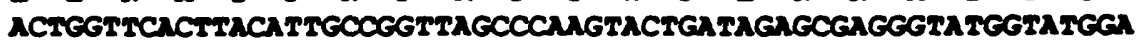

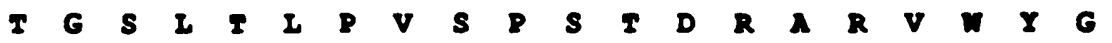

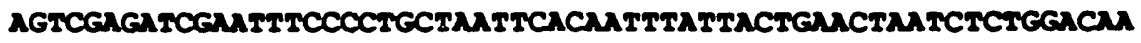
$\begin{array}{llllllllllllllllllll}S & R & D & R & I & S & P & A & N & S & Q & F & I & I & I & I & I & S & G & 0\end{array}$

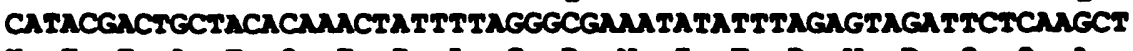

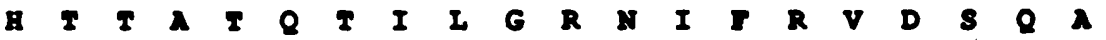

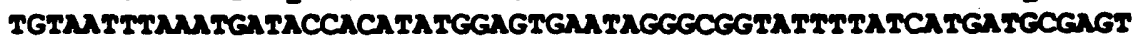

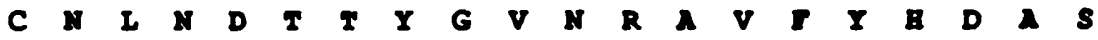
GNAGTTCTCNMGATCCGTGTACGAGGGTATATTCGNACNACTGGGATגGAтגACCT

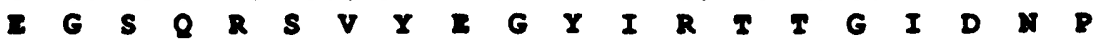

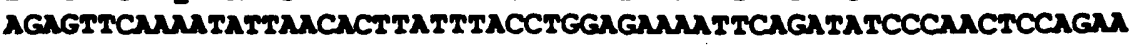

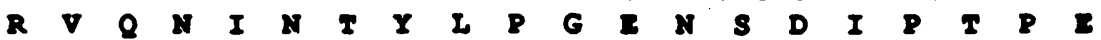

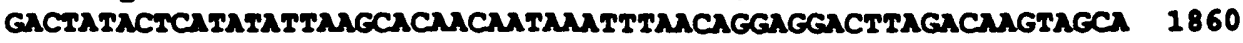
$D \quad I \quad I \quad I \quad I \quad I \quad S \quad I \quad I \quad I \quad I \quad I \quad I \quad G \quad G \quad I \quad R \quad Q \quad V \quad A$

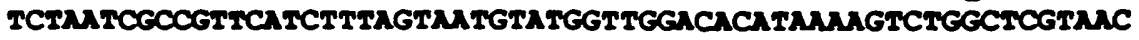

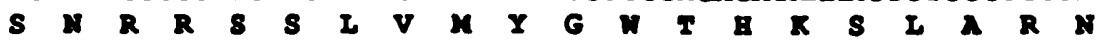

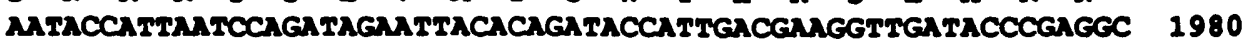

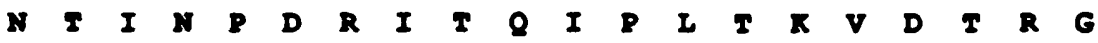
ACAGGTGTTCTRATGTGATGATCCAGGATTATAGGAGAGCTCTACTTCANAGGACT 2040

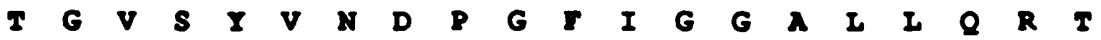


GACCATGGTTCGCTTGGATATTGAGGGTCANTTTCCACTTCACTTMAGACACAMTAT 2100 $\begin{array}{llllllllllllllllllll} & B & G & S & I & G & V & I & R & V & Q & I & P & I & A & I & R & Q & Q & Y\end{array}$

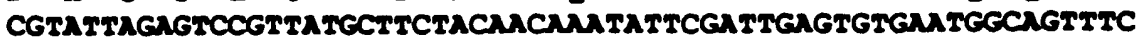
$\begin{array}{llllllllllllllllllll}R & I & R & V & R & Y & A & 8 & I & I & N & I & R & I & S & V & N & G & 8 & \boldsymbol{T}\end{array}$ GGTACTATTICICNANATCTCCCTAGTACNATGAGATIAGGAGAGGATTRAGATACGGA 2160 $\begin{array}{llllllllllllllllllll}G & I & I & S & 9 & A & I & P & S & T & A & R & I & G & I & D & I & R & Y & G\end{array}$

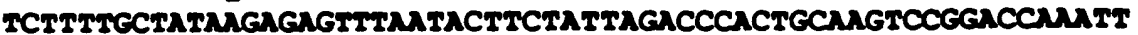

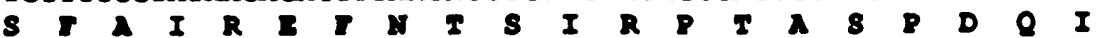

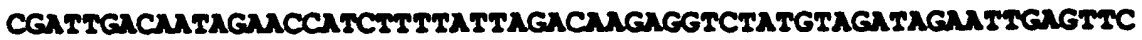

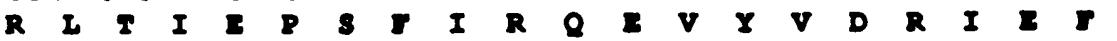

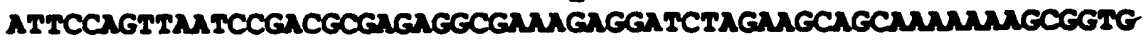

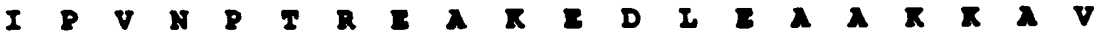

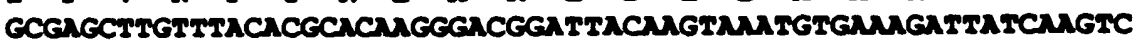
$\begin{array}{llllllllllllllllllll}A & S & I & F & I & R & T & R & D & G & I & Q & V & N & V & X & D & I & Q & V\end{array}$

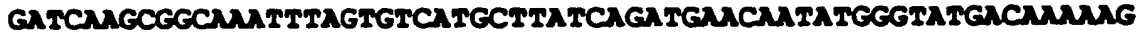

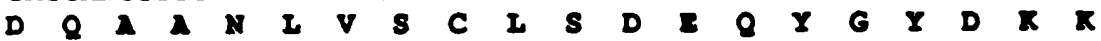

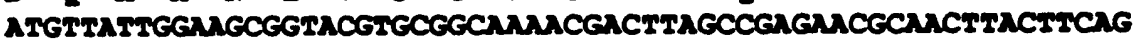
$\begin{array}{llllllllllllllllllll} & I & I & I & A & V & R & A & A & R & R & I & S & R & I & R & A & I & I & Q\end{array}$

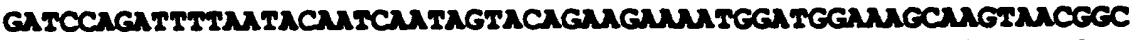

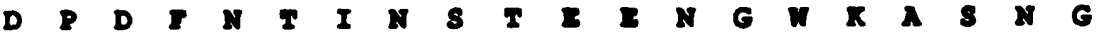

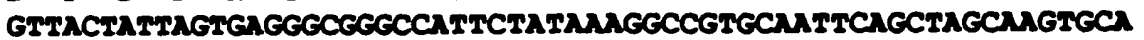

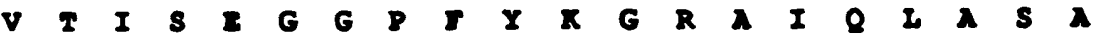

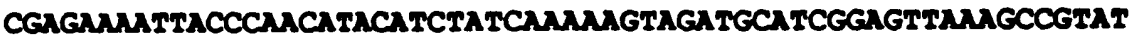

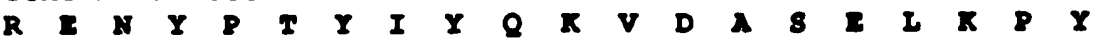

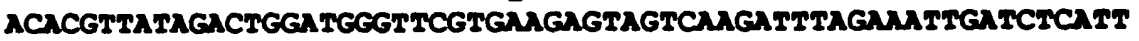

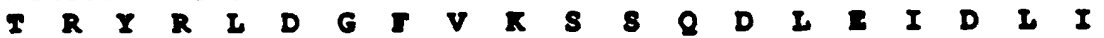

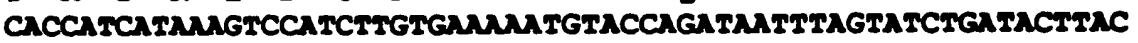
$\begin{array}{llllllllllllllllll}A & B & B & V & B & I & V & X & V & V & D & N & I & V & S & D & I & Y\end{array}$

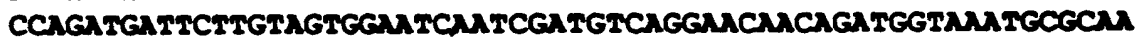
$\begin{array}{llllllllllllllllllll}P & D & D & S & C & S & G & I & N & R & C & Q & \mathbf{I} & Q & Q & M & V & A & A & Q\end{array}$

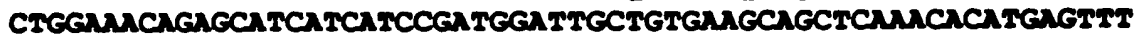

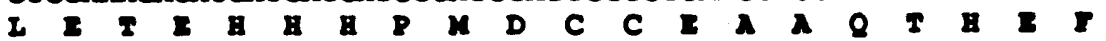

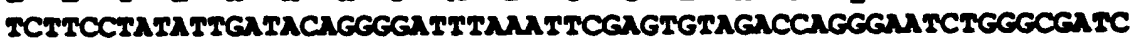

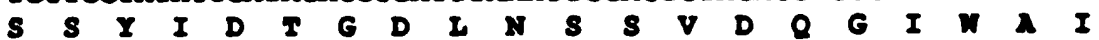
TTPNAGTICGNCACCGATGTPACCGACGTIAGGNATCTTGNATGGTAGAGGTC

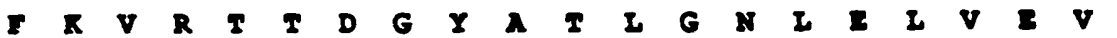

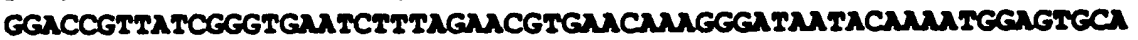

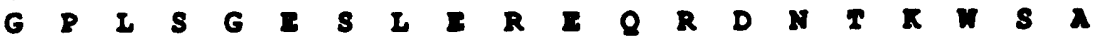
GAGCTAGGNGNAGCGTGCAGNACAGATCGCGTGTATCNAGATGCCNACNATCCATC $\begin{array}{llllllllllllllllllll} & I & G & R & R & R & A & E & I & D & R & V & Y & Q & D & A & \pi & 0 & S & I\end{array}$

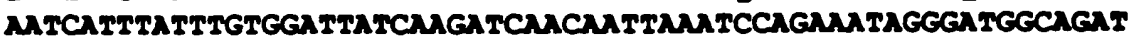

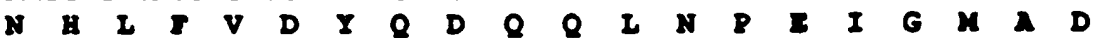

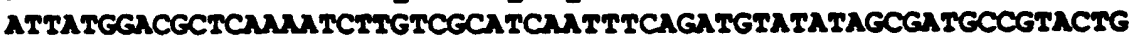

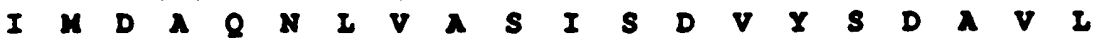

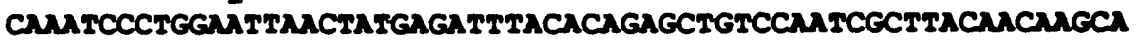

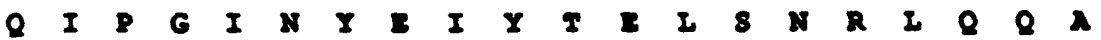

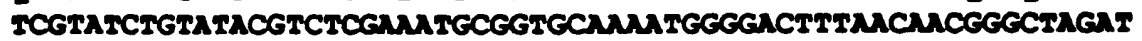
$\begin{array}{llllllllllllllllllll}S & I & I & I & I & S & R & H & A & V & Q & N & G & D & I & A & N & G & I & D\end{array}$

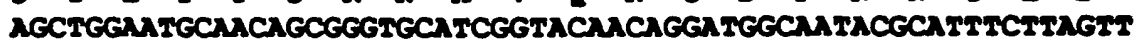

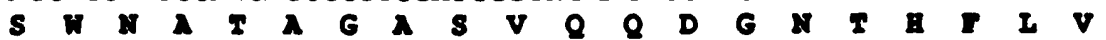

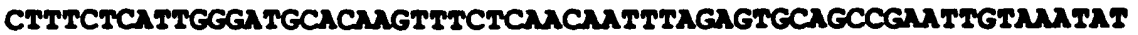

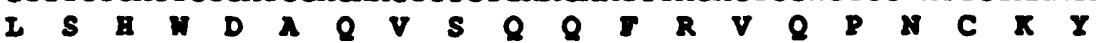

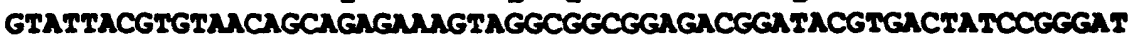
$\begin{array}{llllllllllllllllllll}V & I & R & V & I & A & E & K & V & G & G & G & D & G & Y & V & I & I & R & D\end{array}$

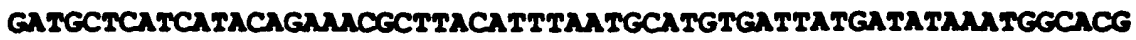

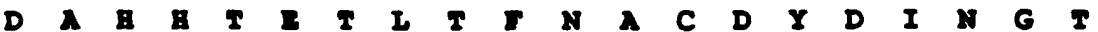

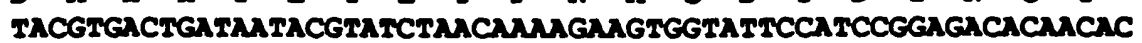

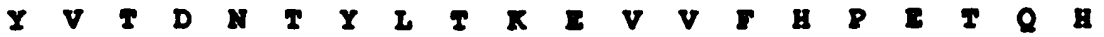
ATGTGGGTAGAGGTMATGNACAGMGGTGCATTTCATATAGATAGTATrGNATTC 


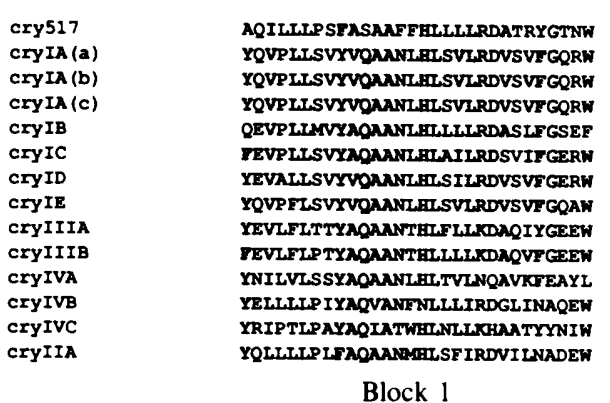

Block 1

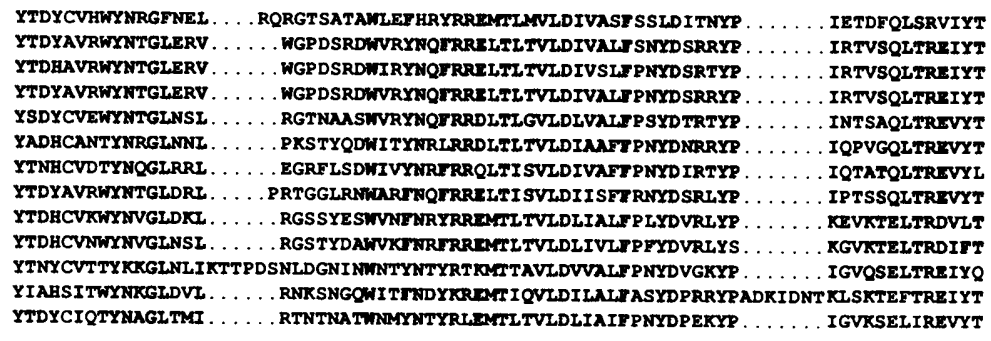

Block 2

\begin{tabular}{|c|c|}
\hline \multirow[t]{2}{*}{$\begin{array}{l}\text { cry517 } \\
\text { cryIA (a) } \\
\text { cryIA (b) } \\
\text { cryIA (c) } \\
\text { cryIB } \\
\text { cryIC } \\
\text { cryID } \\
\text { cryIE } \\
\text { cryIIIA } \\
\text { cryIIIB } \\
\text { cryIVA } \\
\text { cryIVB } \\
\text { cryIVC }\end{array}$} & 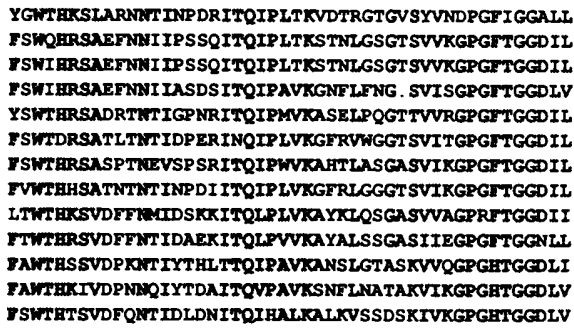 \\
\hline & Block 3 \\
\hline
\end{tabular}

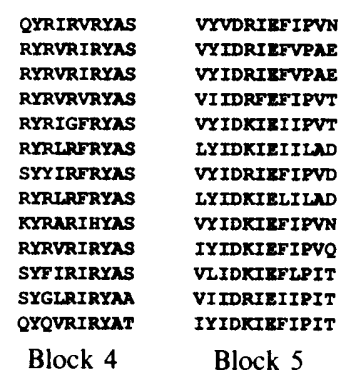

Fig. 3. Comparison of the conserved amino acid sequence blocks encoded by the $c r y-517$ gene with those encoded by other $c r y$ genes. Amino acids marked by the shading are identical or conservatively changed for at least 10 of the 13 sequences. References for sequences can be found in Hofte \& Whiteley (1989), except for cryIE (Visser et al., 1990) and cryIIIB (Sick et al. 1990).

No significant homology was found between sequences upstream of the cry-517 translational start and the proposed promoter sequences for previously reported $B$. thuringiensis cry genes (Wong et al., 1983; Brown \& Whiteley, 1988, 1990). The absence of such sequences suggests that expression of the cry-517 gene requires alternative RNA polymerase-associated $\sigma$ factors to those used by RNA polymerase when transcribing the cry genes published to date. Alternatively, the cry-517 gene may be expressed as a downstream region of an operon. Although the possibility of the cry-517 gene being expressed as an operon cannot be discounted, the absence of any significant ORF in the $385 \mathrm{bp} 5^{\prime}$ to the ATG start of the cry-517 gene suggests this is unlikely.

\section{Conserved amino acid sequences of Cry 517}

The deduced amino acid sequence of the cry-517 gene shows the presence of the five highly conserved blocks $\left(C_{1-5}\right)$, which are present in the toxic portion of all published $B$. thuringiensis Cry proteins, with the exception of Cry II and Cry IV D (Hofte \& Whiteley, 1989). The conserved blocks of the Cry 517 protein are located at amino acid residues $185-215\left(\mathrm{C}_{1}\right), 237-306\left(\mathrm{C}_{2}\right)$, 503-550 $\left(\mathrm{C}_{3}\right)$, 571-580 $\left(\mathrm{C}_{4}\right)$ and 645-656 $\left(\mathrm{C}_{5}\right)$. Fig. 3 shows an alignment of the conserved blocks of representatives of each of the classes of $B$. thuringiensis Cry proteins with those of Cry 517. Comparison of the amino acid sequence of these aligned blocks indicates that $\mathrm{C}_{1}$, $\mathrm{C}_{2}$ and $\mathrm{C}_{3}$ of Cry 517 show $54-70 \%$ similarity (identical and conservative amino acid changes) to Cry I and Cry III proteins and only $46-57 \%$ similarity to Cry IVA-C proteins. The relatively shorter $\mathrm{C}_{4}$ and $\mathrm{C}_{5}$ blocks show a high degree of similarity $(>70 \%)$ to all published Cry I, III and IVA-C protein sequences.

The overall pattern of amino acid similarity between Cry proteins is maintained by Cry 517. There are however, a number of amino acids in the conserved blocks which are conserved among the published Cry proteins but not conserved in Cry 517 nor are they altered to similar amino acids. One such difference occurs in block $C_{1}$, four in $C_{2}$ and four in $C_{3}$. Blocks $C_{1}$ and $C_{2}$ are thought to be involved directly in toxicity, whereas $\mathrm{C}_{3-5}$ are indirectly involved in host specificity (Wu \& Aronson, 1990). Because of these differences in the conserved blocks, it is conceivable that the Cry 517 protein may have considerably different toxicity levels and host spectrum from other Cry proteins. Detailed analysis of the host spectrum and levels of toxicity of the cry-517 gene product is required to establish the significance of these variations.

The hydrophobicity profile of the Cry 517 amino acid sequence indicates a hydrophobic region upstream of the $C_{1}$ block between amino acid residues 57-94. This hydrophobic region is characteristic of Cry proteins, and probably spans the membrane of insect midgut epithelial cells, as has been proposed for the corresponding hydrophobic region of the Cry IA(a) protein (Schnepf $e t$ al., 1985). 


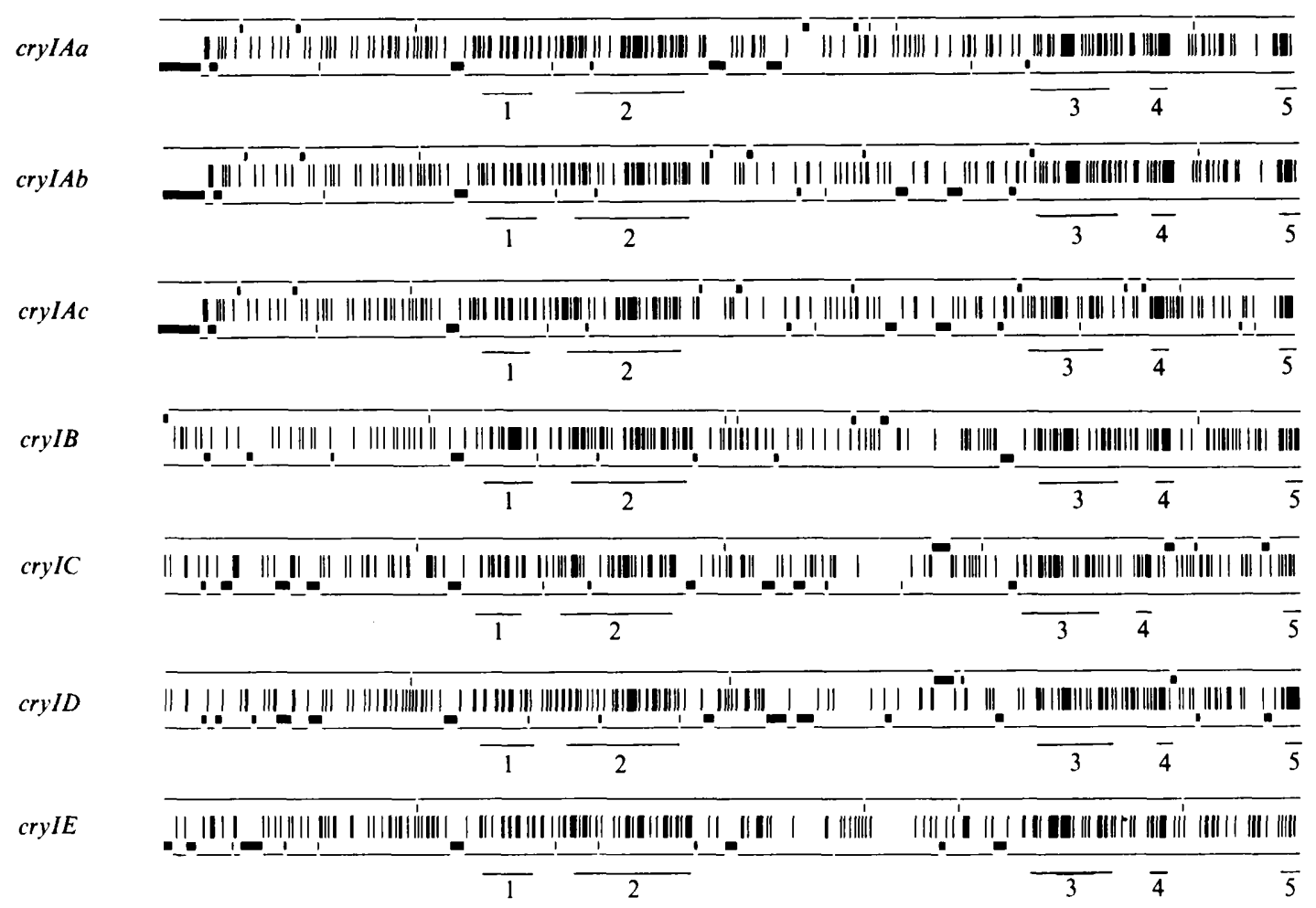

Fig. 4. Amino acid sequence comparisons of the cry-517 gene product with those of other $c r y I$ genes. The comparisons extend from the $\mathrm{N}$-terminus of the protein through to the end of the conserved block $\mathrm{C}_{5}$. The top line represents the Cry 517 amino acid sequence, the bottom line the Cry amino acid sequence with which it is being compared, as listed to the left of the diagram. Vertical lines represent perfectly conserved amino acids between the two sequences. Gaps introduced to obtain maximum alignment are represented by blocks above and below the vertical lines. The locations of conserved amino acid blocks (1-5) from Fig. 3 are indicated.

In addition, five potential trypsin cleavage sites, which could act as the proteolytic cleavage sites necessary for activation of the protoxin in the insect midgut, are located in and around block $\mathrm{C}_{5}$, between amino acid residues $649-670$.

\section{Alignment of Cry 517 protein sequence with other Cry I proteins}

Alignment of the Cry 517 amino acid sequence with other Cry proteins indicates that Cry 517 is more closely related to Cry IA-E, showing 37-39\% homology throughout the entire sequence, than to Cry II $(23 \%)$, Cry III $(30 \%)$ or Cry IVA-C (33-34\%). Fig. 4 shows a diagrammatic representation of the homology between Cry 517 and Cry IA-E proteins up to the end of the block $\mathrm{C}_{5}$ (i.e. the minimal toxic portion of the protein). The level of homology throughout this segment is $29-31 \%$, whereas the homology beyond this region to the C-terminus is $43-45 \%$. Optimal alignment of the C-terminal portion of the Cry 517 protein (amino acid residues 657-1156) with Cry IA-E proteins requires the introduction of two significant gaps into the Cry 517 sequence suggesting a deletion of some 68 amino acids. This is similar to the deletion of 26 amino acids observed when aligning the C-terminal portion of Cry IA(b) with other Cry I proteins (Geiser et al. 1986).

Amino acid conservation between Cry 517 and other Cry I proteins extends throughout the molecule, but as previously discussed, is significantly higher in the regions of the five conserved blocks and the C-terminal region of the protein, which is cleaved off during protoxin activation. A high degree of heterogeneity occurs in the regions between the conserved blocks, most significantly between $\mathrm{C}_{2}$ and $\mathrm{C}_{3}$ which shows less than $22 \%$ identity to other Cry I proteins. This region has been implicated in the determination of host specificity of several Cry proteins (Ge et al., 1989; Schnepf et al. 1990).

The N-terminal region of the Cry 517 protein differs from other Cry I proteins in containing a considerably greater number of amino acids in the region between the initial methionine and the $\mathrm{C}_{1}$ block. However this may be of little significance as it has been demonstrated that 
part of the $\mathrm{N}$-terminus of Cry I proteins is not required for insecticidal activity (Hofte et al., 1986). The important feature of the $\mathrm{N}$-terminal region of the protein is probably the spatial relationship between the hydrophobic region and $\mathrm{C}_{1}$ block. The number of amino acids between these two regions of the Cry 517 protein approximates to that of other Cry I proteins suggesting that the spatial relationship is maintained.

Although the $c r y-517$ gene appears to have at least a distant ancestral relationship to other $c r y I$ genes there are no significantly long stretches of homology to suggest that part of the gene has a closer evolutionary relationship to one cryI gene rather than another or, as is suggested for $c r y I E$ (Visser et al., 1990), that $c r y-517$ is a result of a recombination event between two known cry genes.

\section{Insecticidal activity of the cry-517 gene product}

Western blot analysis of the Cry 517 protein from recombinant $\lambda \mathrm{gtll}$ lysates indicated a low level of expression of the $c r y-517$ gene in E. coli. This may have been due to the lack of an $E$. coli promoter sequence. Alternatively, the low level expression may have been due to the absence of a transcriptional terminator, $3^{\prime}$ to the $c r y-517$ ORF, resulting in unstable cry-517 mRNA transcripts. The latter explanation has been observed with other genes lacking transcriptional terminators (Wong \& Chang, 1985). Due to this low level of expression of the Cry 517 protein in E. coli, some difficulty was encountered in obtaining adequate protein for extensive toxicity studies. However, preliminary bioassays showed that $100 \mu \mathrm{l}$ of recombinant $\lambda \mathrm{gt} 11$ lysate incorporated into $1 \mathrm{ml}$ of artificial diet (Malone \& Wigley, 1990) caused $70 \%$ mortality of first instar larvae of Epiphyas postvittana (Lepidoptera: Tortricidae) in $6 \mathrm{~d}$, whereas controls showed less than $10 \%$ mortality, demonstrating that the recombinant Cry 517 protein is biologically active.

The estimated molecular mass of $129700 \mathrm{Da}$, and the fact that the amino acid sequence appears more closely related to Cry I proteins than to Cry II, III or IV proteins, suggests that according to the Hofte \& Whiteley (1989) classification system, the $c r y-517$ gene is a cryI-type but is sufficiently different to form a new subclass. Although further characterization of the gene product, particularly toxicity data, is required before this gene can be assigned a $c r y$ number, we tentatively refer to the $c r y-517$ gene as cryIG.

We are grateful to D. Christie (University of Auckland) for the $\mathrm{N}$-terminal amino acid determination and to R. L. S. Forster for his critical reading of this manuscript.

\section{References}

Brown, K. L. \& Whiteley, H. R. (1988). Isolation of a Bacillus thuringiensis RNA polymerase capable of transcribing crystal protein genes. Proceedings of the National Academy of Sciences of the United States of America 85, 4166-4170.

BROWN, K. L. \& WHITELEY, H. R. (1990). Isolation of a second Bacillus thuringiensis RNA polymerase that transcribes from a crystal protein gene promoter. Journal of Bacteriology 172, 6682-6688.

Del Sal, G., Manfioletta, G. \& Schneider, C. (1988). A one tube plasmid DNA minipreparation suitable for sequencing. Nucleic Acids Research 16, 9878.

Ge, A. Z., Shivarova, V. I. \& Dean, D. H. (1989). Location of the Bombyx mori specificity domain on a Bacillus thuringiensis $\delta$ endotoxin protein. Proceedings of the National Academy of Sciences of the United States of America 86, 4037-4041.

Geiser, M., SChweitzer, S. \& Grimm, C. (1986). The hypervariable region in the genes coding for entomopathogenic crystal proteins of Bacillus thuringiensis: Nucleotide sequence of the kurhdl gene of subsp. kurstaki HDl. Gene 48, 109-118.

HOFTE, H. \& WhITELEY, H. R. (1989). Insecticidal crystal proteins of Bacillus thuringiensis. Microbiological Reviews 53, 242-255.

Hofte, H., de Greve, H., Seurinck, J., Jansens, S., Mahillon, J., AMP, C., VanderkerckhoVe, J., VanderbrugGen, H., VaN MONTAGU, M. \& VAECK, M. (1986). Structural and functional analysis of a cloned delta endotoxin of Bacillus thuringiensis Berliner 1715. European Journal of Biochemistry 161, 273-280.

MCGAUGHEY, W. H. (1985). Insect resistance to biological insecticide Bacillus thuringiensis. Science 229, 193-195.

MCGaughey, W. H. \& Beeman, R. W. (1988). Resistance to Bacillus thuringiensis in colonies of Indian meal moth and Almond moth (Lepidoptera: Pyralidae). Journal of Economic Entomology 81, 28-33.

MALONE, L. A. \& Wigley, P. J. (1990). A practical method for rearing Argentine Stem Weevil, Listronotus bonariensis, (Coleoptera: Curculionidae) in the laboratory. New Zealand Entomologist 13, 87-88.

Maniatis, T., Fritsch, E. F. \& Sambrook, J. (1982). Molecular Cloning: a Laboratory Manual. Cold Spring Harbor, NY: Cold Spring Harbor Laboratory.

MatSuDAiRA, P. (1987). Sequence from picomole quantities of proteins electroblotted onto polyvinylidene difluoride membranes. Journal of Biological Chemistry 262, 10035-10038.

Meyer, R., Figurski, D. \& Helinski, D. R. (1977). Physical and genetic studies with restriction endonucleases on the broad-host range plasmid RK2. Molecular and General Genetics 152, 129-135.

SANGer, F., Nicklen, S. \& Coulson, A. R. (1977). DNA sequencing with chain-terminating inhibitors. Proceedings of the National Academy of Sciences of the United States of America 74, 5463-5467.

SCHNEPF, H. E., Wong, H. C. \& Whiteley, H. R. (1985). The amino acid sequence of a crystal protein from Bacillus thuringiensis deduced from the DNA base sequence. Journal of Biological Chemistry 260, 6264-6272.

Schnepf, H. E., Tomczak, K., Ortega, J. P. \& Whiteley, H. R. (1990). Specificity-determining regions of a lepidopteran-specific insecticidal protein produced by Bacillus thuringiensis. Journal of Biological Chemistry 265, 20923-20930.

Sick, A., GaERTner, F. \& Wong, A. (1990). Nucleotide sequence of a coleopteran-active gene from a new isolate of Bacillus thuringiensis subsp. tolworthi. Nucleic Acids Research 18, 1305.

Stewart, G. S. A. B., Johnston, K., Hagelberg, E. \& Ellar, D. J. (1981). Commitment of bacterial spores to germinate. A measure of the trigger reaction. Biochemical Journal 196, 101-106.

TABashnik, B. E., Cushing, N. L., Finson, N. \& Johnson, M. W. (1990). Field development of resistance to Bacillus thuringiensis in Diamondback moth (Lepidoptera: Plutellidae). Journal of Economic Entomology 83, 1672-1676.

Van Rie, J., McGaughey, W. H., Johnson, D. E., Barnett, B. D. \& Van MellaerT, H. (1990). Mechanism of insect resistance to the microbial insecticide Bacillus thuringiensis. Science 247, 72-74. 
Visser, B., Munsterman, E., Stoker, A. \& Dirkse, W. G. (1990). A novel Bacillus thuringiensis gene encoding a Spodoptera exiguaspecific crystal protein. Journal of Bacteriology 172, 6783-6788.

WIGLEY, P. J. \& CHILCOTT, C. N. (1990). Bacillus thuringiensis isolates active against the New Zealand pasture pest, Costelytra zealandica (Coleoptera: Scarabaeidae). In Proceedings of the Vth International Colloquium on Invertebrate Pathology and Microbial Control, p. 344. Edited by D. E. Pinnock. Adelaide.

WOLFERSBERGER, M. (1990). Specificity and mode of action of a Bacillus thuringiensis insecticidal crystal protein to lepidopteran larvae: Recent insights from studies using midgut brush border membrane vesicles. In Proceedings of the Vth International Colloguium on Invertebrate Pathology and Microbial Control, pp. 278-282. Edited by D. E. Pinnock. Adelaide.

Wong, H. C. \& ChANG, S. (1985). Identification of a positive retroregulator that functions in Escherichia coli and Bacillus subtilis. In Molecular Biology of Microbial Differentiation, pp. 104-109. Edited by J. A. Hoch \& P. Setlow. Washington, DC: ASM.

WoNG, H. C., Schnepf, H. E. \& Whiteley, H. R. (1983). Transcriptional and translational start sites for the Bacillus thuringiensis crystal protein. Journal of Biological Chemistry 172, $6682-6688$.

WU, D. \& ARONSON, A. I. (1990). Use of mutagenic oligonucleotides for defining regions of Bacillus thuringiensis $\delta$ endotoxin involved in toxicity. In Proceedings of the Vth International Colloquium on Invertebrate Pathology and Microbial Control, pp. 273-277. Edited by D. E. Pinnock. Adelaide.

Yanisch-Perron, C., Vieira, J. \& Messing, J. (1985). Improved M13 phage cloning vectors and host strains: nucleotide sequence of the M13mp18 and pUC19 vectors. Gene 33, 103-119. 